\title{
P-Carbon CNI Polarimeter Operation Experience
}

\author{
H. Huang ${ }^{*}$, A. Poblaguev, D. Steski, K. Yip, A. Zelenski \\ Brookhaven National Laboratory, Upton, NY 11973, USA \\ E-mail: huanghai@bnl.gov
}

\begin{abstract}
The p-carbon polarimeter working in the dp/p Coulomb Nuclear Interference (CNI) region has been used in the Brookhaven Alternating Gradient Synchrotron (AGS ) for many years. It has gone though several upgrades to improve its performance. With targets sweeping through beam vertically and horizontally, the polarimeter can provide polarization profile information. To preserve polarization, a pair of horizontal tune jump quads have been used to overcome horizontal intrinsic resonances. Recently, the polarization measurement on the energy ramp has been used to decide the beam energy as function of time on the ramp. This paper summarize the recent operation experience of AGS p-carbon CNI polarimeter.
\end{abstract}

XVII International Workshop on Polarized Sources, Targets \& Polarimetry

16-20 October 2017

Kaist, South Korea

* Speaker.

$\dagger$ This document has been authorized by employees of Brookhaven Science Associates, LLC under Contract No. DE-SC0012704 with the U.S. Department of Energy. The United States Government retains a non-exclusive, paid-up, irrevocable, world-wide license to publish or reproduce the published form of this document, or allow others to do so, for United States Government purposes. 


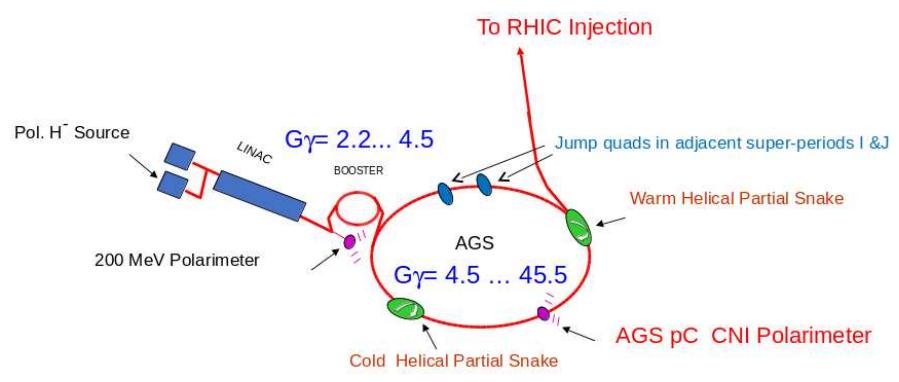

Figure 1: The general layout of polarized proton apparatus in the injectors of RHIC.

\section{Introduction}

AGS has been running as polarized proton injector for Relativistic Heavy Ion Collider (RHIC) with dual partial snakes [1] and two horizontal tune jump quads [2] since 2011. The dual partial snakes overcome the vertical intrinsic and imperfection depolarizing resonances in the AGS. The introduction of partial snakes generates horizontal intrinsic resonances. They are generally weak but could cause accumulated polarization loss if left uncorrected [3]. A modest horizontal tune jump system has been used to overcome these weak but numerous resonances while maintaining the transverse emittances. A relative gain of $10-15 \%$ polarization has been achieved with the tune jump system. The general layout of polarized proton apparatus of RHIC injectors are shown in Fig. 1. A fast polarimeter is cirtical for the polarized proton setup and polarization maintenance. This paper summarize the operation experience of the polarimeter.

\section{Coulomb-Nuclear Interference}

The elastic $p$-Carbon scattering at Coulomb-nuclear interference (CNI) region is the process used to measure polarization. Small angle elastic scattering of hadrons in the CNI region has long been advocated for polarimetry. The predicted asymmetry is significant and largely independent of energy for energy above a few GeV. For $p$-Carbon CNI, the protons scattered forward are not easily detectable (it stays within the beam), and the energy of the recoil carbon nucleus is $100-600 \mathrm{keV}$. The low energy carbon would stop in most targets. The $p$-Carbon CNI polarimeter becomes feasible with the development of very thin ribbon carbon targets at IUCF[4]. The slowness of the recoil carbon also makes detection difficult. However, the arrival time of the carbon can be set to be in between bunches, avoiding prompt background. Fig. 2 shows the Carbon events detected by the Si detector. The heavy concentration part in a banana shape is the recoil carbons from p-Carbon CNI region.

\section{Polarimeter Configuration}

We employ silicon detectors for the detection of recoil carbon ions from the $\vec{p}+C$ elastic scattering. A vacuum chamber hosts the detectors and target ladders. The carbon target in use is carbon micro-ribbons of $30 \mathrm{~nm}$ thickness, with a typical width of $50 \mu \mathrm{m}$ and $5.0 \mathrm{~cm}$ long. There 


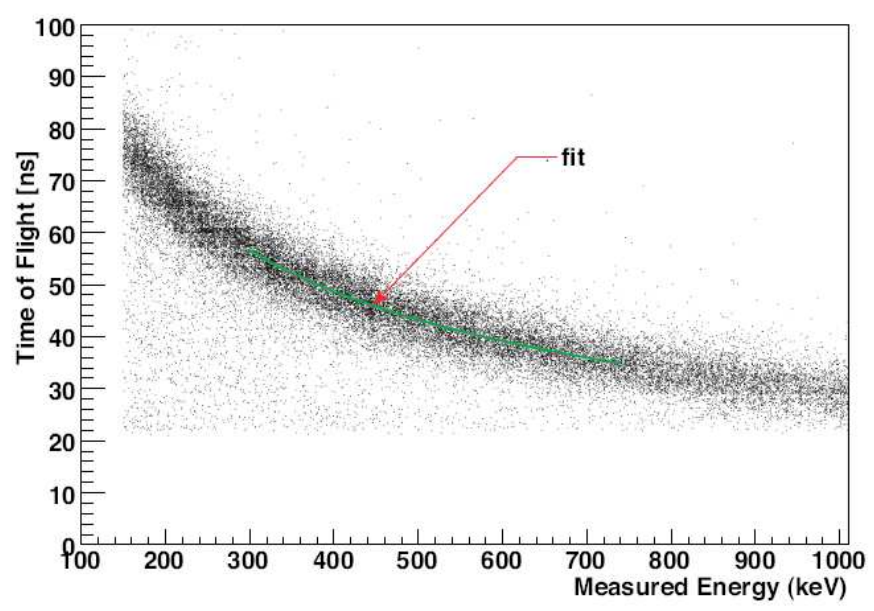

Figure 2: The events in the Time-of-Flight vs Energy plot.

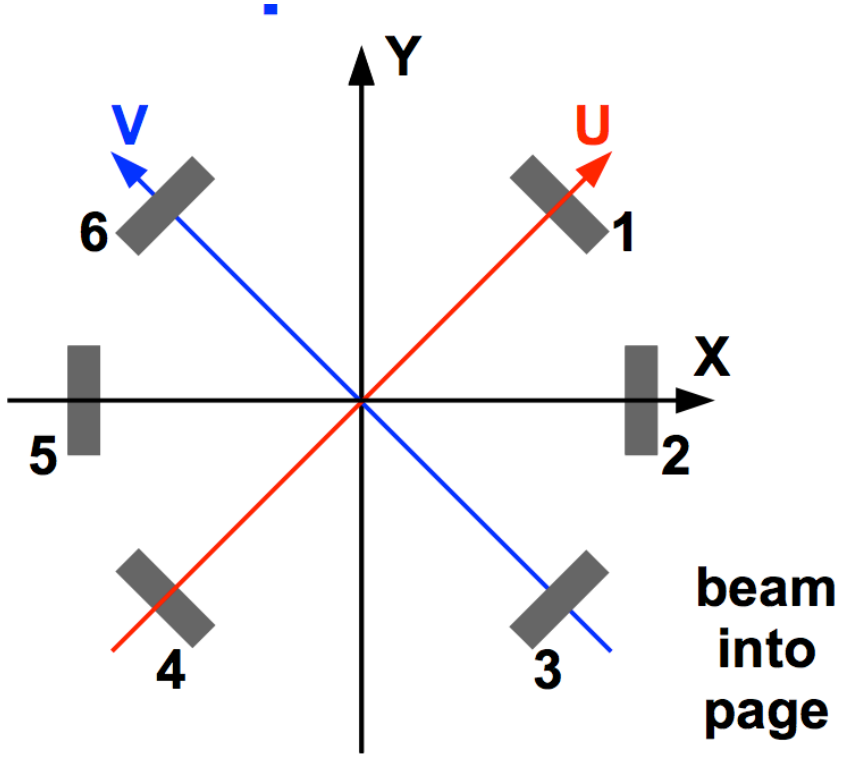

Figure 3: There are three pairs of detectors. The pairs at $\pm 45^{\circ}$ measure both horizontal and vertical polarization components. The pair at $90^{\circ}$ measures vertical component only.

are also several targets with different widths: $75 \mu \mathrm{m}$ to $125 \mu \mathrm{m}$ for event rate study. The thin target makes the measuring process almost a non-destructive process to polarized beam. The manufacturing process for such ribbons has been developed at IUCF [4], and is now routine at BNL[5]. The ribbons are mounted perpendicular to the beam direction in free suspension between the ends of a fork. The fork is moved into the beam in every AGS spill. Even though the target life time is months long, we still need spares for replacement. To study the rate effect, different target sizes are needed. To measure polarization profiles in both horizontal and vertical planes, target orientation in both planes are required. As the results, there are 8 carbon targets (4 vertical and 4 horizontal). Four targets can be mounted on each target ladder. 


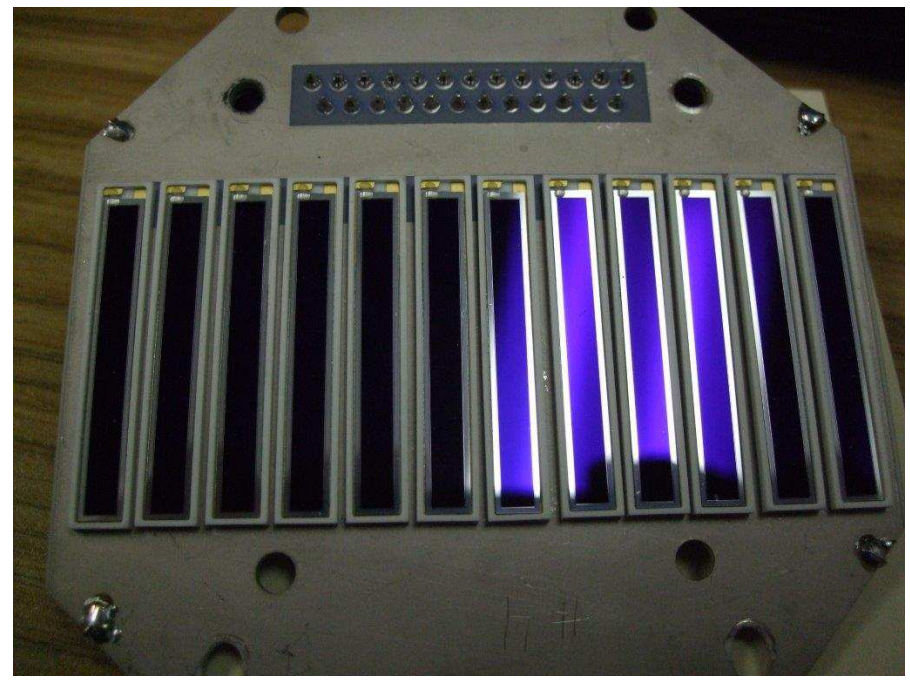

Figure 4: One detector consists of twelve Hamamatsu strip detectors mounted on one ceramic board.

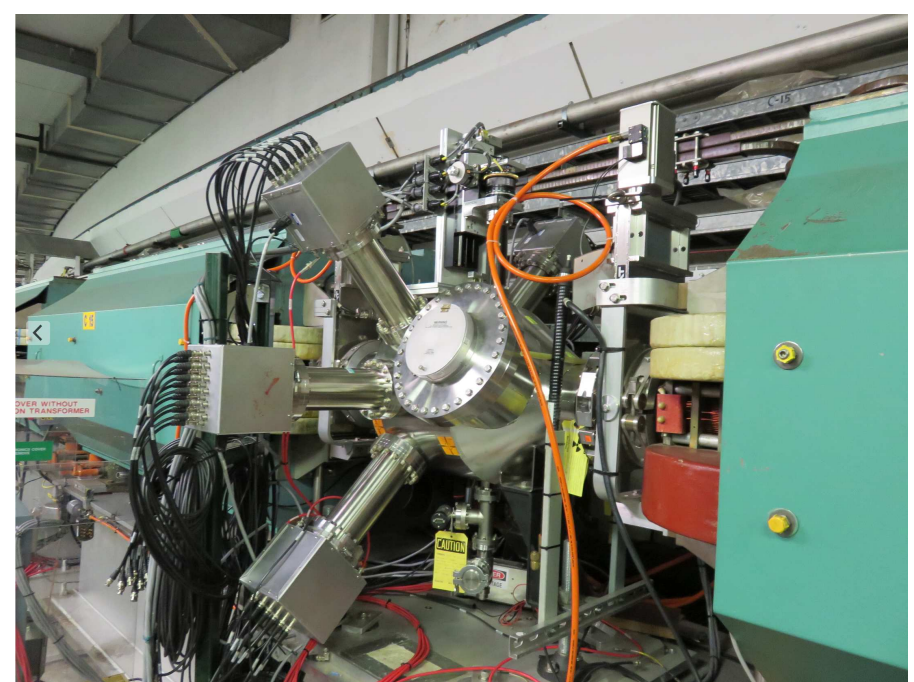

Figure 5: The assembly of AGS CNI polarimeter. The extended beam pipes extend the distance of Si detectors to the target from $32 \mathrm{~cm}$ to $51 \mathrm{~cm}$. The aluminum boxes cover the front-end electronics.

There are three pair detectors (see Fig. 3) and each pair is an independent polarimeter. The two detector pairs in the $\pm 45^{\circ}$ measures both vertical and horizontal components of polarization while the detector pair in the $90^{\circ}$ measures vertical polarization only. Each detector consists of twelve Hamamatsu $3 \mathrm{~cm} \times 3 \mathrm{~mm}$ strip detectors mounted on one ceramic board as shown in Fig. 4. Si detector strip orientation is along the beam direction for evenly distributed events over all detector strips. For each Si detector, an Am source is mounted on the opposite side of the chamber for calibration purpose. To reduce the rf noise from beam on the Si detectors, an $\mathrm{rf}$ mesh with $95 \%$ transmission was used to separate the Si detectors from beam.

The whole polarimeter assembly is shown Fig. 5. The purpose of the extension pipes for a target to detector distance of $51.3 \mathrm{~cm}$ is two folds: to have better time of flight resolution; and to 


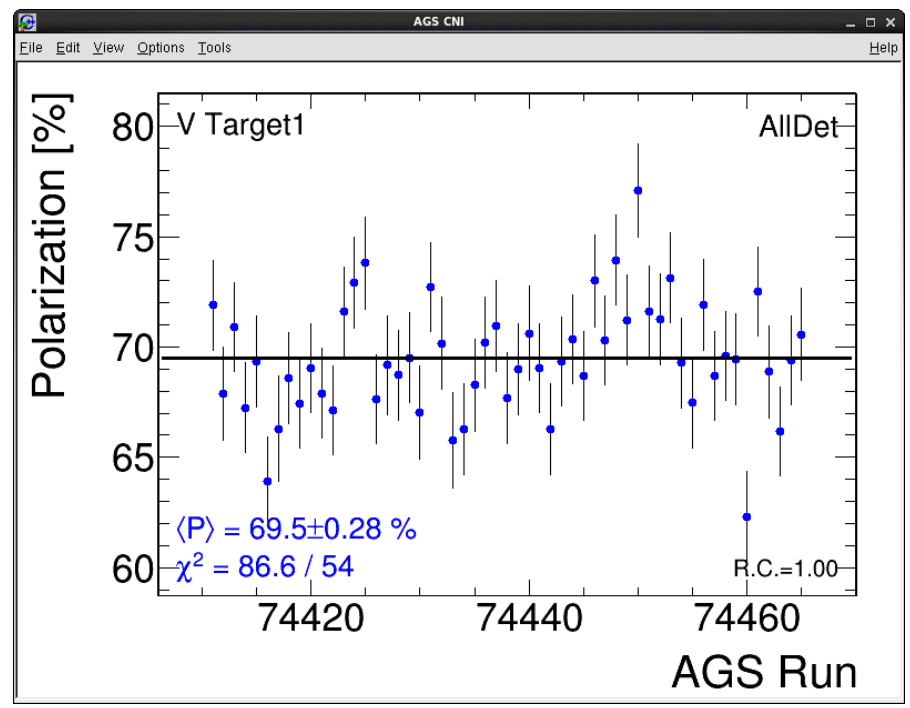

Figure 6: The consecutively measured polarization over several hours. It shows the statistical stability of the polarimeter.

have less event rate after increased the detector size. The constraints are the available space around the polarimeter and the bunch spacing.

The preamplifiers are installed outside the vacuum and the signals then travel 300 feet to the DAQ electronics. Since the noise is significantly high around AGS ring, special efforts are taken to reduce the noise from rf. The bias voltage power supply for the Si detectors is in the ring next to the vacuum chamber. An aluminum cage covers preamplifiers and reduces pick-up noise significantly. The analog signals are also differentiated before digitized to eliminate rf noise.

\section{Polarimeter Operation}

The high event rate, $1 \mathrm{M}$ events/sec at top energy with no dead time provides fast measurement time with small statistical error. A relative error of 3\% in $\mathrm{dP} / \mathrm{P}$ can be achieved in 3 minute measurement time. The analyzing power of the p-Carbon CNI polarimeter was measured with extracted beam on a carbon target where both forward and recoil particles can be identified [6]. The stability of the polarimeter has been tested with consecutive polarization measurements over several hours for the same beam conditions. The results are shown in Fig. 6. With targets sweeping through beam vertically and horizontally, the polarimeter can provide polarization profile information. These information are important to assess the polarization of the proton beam.

Besides polarization measurement at fixed energy, the polarimeter can also measure asymmetry on the energy ramp. One example is shown in Fig 7. Measured asymmetry reflects predicted spin dynamics. With partial Siberian snake in the AGS ring, spin direction flips at $G \gamma=$ integer, where $G$ is anormalous magnetic moment of proton, $\gamma$ is Lorentz factor. Decreasing amplitude with $G \gamma$ due partly to polarization loss, mostly to reduction of $p C$ analyzing power. Such a measurement can be used to determine the beam energy accurately.

The tune jump quads jump the tune with 0.04 unit in 100us. To get the benefit of the jump quads, we need to get the $70+$ tune jump timing correct down to the level 10-20us. In run15, the 


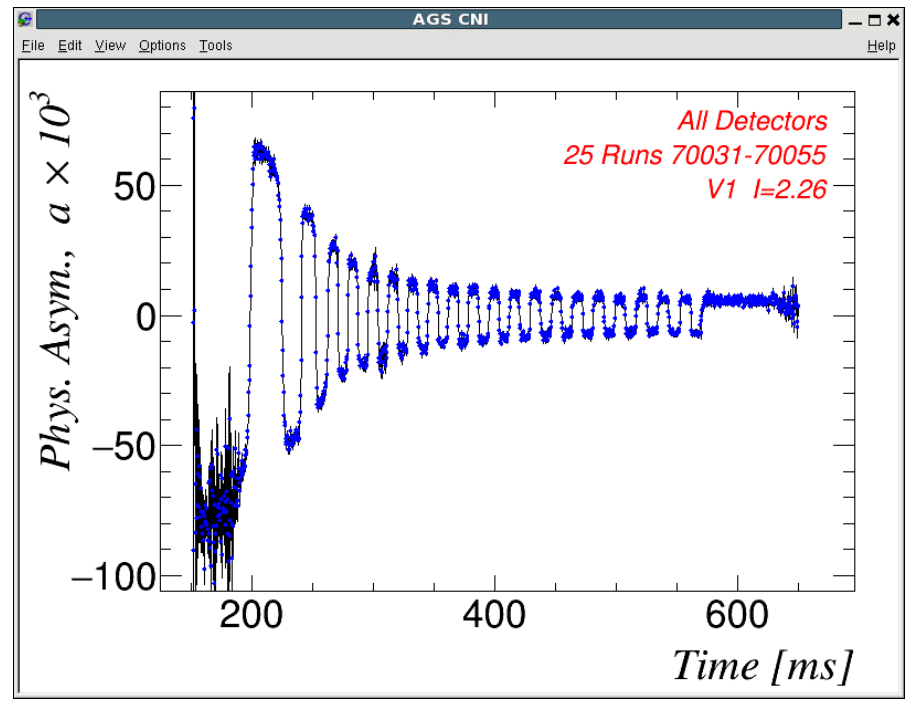

Figure 7: The asymmetry measured on the AGS ramp as function real time. The spin flips at every integer of $G \gamma$ are due to the presence of two partial Siberian snakes.

horizontal tune jump quadrupoles became ineffective from time to time. Many factors can change the jump quadrupole timing, such as AGS main magnet field drift, radial change, and betatron tune change. The required timing accuracy is below $100 \mu \mathrm{s}$, which is challenging. It is critical that the multiple tune jumps happen at the right energies. The horizontal tune jump amplitude of 0.04 is equivalent to about 8 Gauss magnetic field change. It was noticed that the AGS main magnet field measurement drifted over five months operations. The drifts seem to be correlated with the room temperature and cooling water temperature. The AGS main magnet regulation circuitry was modified to reduce the temperature impact. Nevertheless, the B field variation is still observed. So this is likely not the source of the timing drifting. At the beginning of the operation of horizontal tune jump, the timing was derived from betatron tune, main magnet field and beam radius along the ramp. Due to the errors of the last two quantities, the derived tune jump timing could have errors, too.

From the asymmetry measurement on the ramp, the spin flip information can be used to determine the energies and timings of these spin flips. Consequently, the jump quad timing can be derived from these spin flips. In the past, a sophisticated combination of spin flips and B field/radius measurements were used. But it was not good enough to find beneficial jump quad timings after main magnet drifts. Recently, the jump quad timing derivation was modified. Only spin flip information was used to derive jump quad timing. The results are very robust: they show consistent beneficial jump quad timing files while the magnet drift is still present during the five months operation. These results may suggest that the main magnet drift could be just a measurement issue.

\section{Polarization Measurement Accuracy}

By design, the AGS polarimeter is a relative polarimeter. However, with absolute polarization measurement upstream (at end of $200 \mathrm{MeV}$ linac) and downstream (at the RHIC store energy with polarized hydrogen jet), the polarization at AGS extraction can be checked to see how far it is from 
true polarization. Of course, this will rely on the understanding of the polarization loss in the AGS. With dual partial snakes in the AGS, the residual small polarization loss is believed due to only intrinsic depolarizing resonances, which is reflected in the development of polarization profiles. Assuming the polarization profile is similar to beam as Gaussian distribution, the ratio $R$ is given as $R=\left(\sigma_{\text {beam }} / \sigma_{\text {pol }}\right)^{2}$ [7]. From the measurements done in AGS, the ratio $R \sim 0.1$ at flattop for the typical running $2 \times 10^{11}$ bunch intensity. If the development of a polarization profile is the primary reason for the reduction of the average polarization $\langle P\rangle$ then

$$
\langle P\rangle=\frac{P_{0}}{\left(1+R_{x}\right)\left(1+R_{y}\right)}
$$

where $P_{0}$ is zero emittance polarization and $R_{x, y}$ are the horizontal and vertical profile ratio, respectively. From this model, the zero emittance polarization derived from AGS flattop energy measurements should be the same as source polarization except a small factor at AGS injection due to the non-vertical stable spin direction. In other words, the $P_{0}$ may be related to the source polarization $P_{\text {source }}$, measured by the $200 \mathrm{MeV}$ absolute polarimeter [8] with a $1 \%$ systematic error

$$
P_{0} / P_{\text {source }} \approx 0.985
$$

Here the $P_{0}$ is calculated from the vertical and horizontal polarization profiles measured at AGS flattop, as well as the polarization value. The $P_{\text {source }}$ was measured by the $200 \mathrm{MeV}$ absolute polarimeter. The small difference is caused by a non-vertical spin direction at the injection to the AGS and at the flattop.

The profile measurements allows us to make an experimental estimate zero emittance polarization: zero emittance polarization can be obtained from the polarization profile measurements done in the AGS at flattop energy:

$$
P_{0}^{\text {meas }}=\sqrt{P_{\max }^{x} P_{\max }^{y}\left(1+R_{x}\right)^{3 / 2}\left(1+R_{y}\right)^{3 / 2}}
$$

where $P_{\max }^{x, y}$ is the maximum polarization from horizontal and vertical polarization profile fit, respectively. In run17, the measured ratio is

$$
P_{0}^{\text {meas }} / P_{\text {source }}=0.97 \pm 0.02
$$

it shows a very good agreement with Eq.(5.2). This implies that the polarization reported by the AGS polarimeter is close to the true beam polarization.

On the other hand, the RHIC polarization at top energy $(255 \mathrm{GeV})$ was calibrated with polarized hydrogen jet target. The target deformation during measurement done with RHIC p-carbon polarimeter probably caused underestimation of the polarization profile, or $R$ value. Nevertheless, we can use the results for consistency check of measured $P_{0}$ [9]. In run17, the average polarization in RHIC yellow ring is $55.7 \pm 0.2 \%$ from all stores. The average $R$ values for both vertical and horizontal planes in RHIC yellow ring is $0.168 \pm 0.006$. The $P_{0} / P_{\text {source }}$ is expected to be 0.976 after taking into account the spin mismatch at RHIC injection. The measured $P_{0}^{\text {meas }} / P_{\text {source }}$ from yellow ring is $0.922 \pm 0.02$. Even if all the difference is attributed from $P_{0}$ instead of underestimation of $R$ value, the difference is still about $5 \%$. This should be the upper limit on the accuracy of the AGS polarimeter. This implies that the AGS polarization is measured within a few percent accuracy for the actual beam polarization. 


\section{Summary}

AGS CNI polarimeter has become a robust tool for AGS polarized proton setup. The beam intensity has increased due to source improvement and better AGS tuning. The target size has been reduced for reasonable event rate(from $250 \mu \mathrm{m}$ to $50 \mu \mathrm{m}$ ). Rate correction is necessary for polarization measurement. The polarimeter provided polarization profile information for accelerator setup tuning. This is especially important since there is polarization loss in the AGS during acceleration. The polarization measurement on the energy ramp has been used to provide the robust jump quads timing. Although the AGS polarimeter has not been calibrated with absolute polarimeter yet, the consistency check with upstream and downstream polarimeters suggests that the polarimeter measures absolute polarization with a few percent accuracy.

\section{References}

[1] H. Huang, et al., Phys. Rev. Lett. 99, 154801 (2007).

[2] H. Huang, et al., Phys. Rev. ST AB 17, 081001 (2014).

[3] F. Lin, et al., Phys. Rev. ST AB 10, 044001 (2007). p.634.

[4] W.R. Lozowski and J.D. Hudson, Nucl. Instr. Meth. A303, 34 (1991).

[5] W.R. Lozowski et al., Nucl. Instr. Meth. A590, 157(2008).

[6] J. Tojo, et al., Phys. Rev. Lett. 89, 052302 (2002).

[7] W. Fischer and A. Bazilevsky, Phys. Rev. ST AB 15, 041001 (2012).

[8] A. Zelenski, et al., J. Phys. Conf. Ser. 295, 012132 (2011).

[9] A. Zelenski, et al., Proc. of PSTP 2013, 056 (2013). 